\title{
EFEITOS DAS TRANSFORMAÇÕES DE DADOS SOBRE O COEFICIENTE DE VARIAÇÃ̃ ${ }^{1}$
}

\author{
Débora Ferreira da Cunha ${ }^{2}$ \\ José Carlos Seraphin ${ }^{3}$ \\ Francisco José P. Zimmermann ${ }^{4}$
}

\section{RESUMO}

A transformação de dados é um processo auxiliar utilizado junto à análise de variância, para torná-la válida quando uma ou mais de suas hipóteses básicas não se verificam. No entanto, mesmo quando feita corretamente, pode trazer inconvenientes como alteração nos parâmetros analisados. O coeficiente de variação (CV) é um destes parâmetros e apresenta, como desvantagem, grande sensibilidade à transformações de dados. Neste trabalho foi estudado o efeito das transformações de potência presentes na Escala de Transformações de Tukey, sobre o CV, para 110 experimentos do arroz de sequeiro, classificados de acordo com tipo de ensaio e região de cultivo, considerando a variável produção de grãos e suas respectivas transformações. Foram usados os testes de Lilliefors e de Cochran para testar, respectivamente, a distribuição dos dados quanto à normalidade, e a homogeneidade de variância dos tratamentos. As transformações mais eficientes foram a raiz quadrada e a logarítmica, ambas reduzindo os valores do $\mathrm{CV}$, corrigindo a falta de normalidade e/ou a heterogeneidade de variância quando havia necessidade de transforma-

1 Parte da Monografia de Especialização em Estatística do primeiro autor, apresentada na Universidade Federal de Goiás (UFG/GO). Jan/2000.

$2 \mathrm{Na}$ época, pós-graduanda em Estatística, Universidade Federal de Goiás - UFG, Goiânia-GO.

3 Instituto de Matemática e Estatística, Universidade Federal de Goiás - UFG, Caixa Postal 131, Goiânia-GO, Brasil.

4 Embrapa Arroz e Feijão (CNPAF), Caixa Postal 179, Goiânia-GO. 
ção. Em geral os experimentos que apresentaram menores $\mathrm{CV}$ foram os do grupo Ensaio Comparativo Avançado, juntamente com aqueles conduzidos na região Centro-Oeste.

Palvras-chave: transformação de dados, coeficiente de variação, arroz de sequeiro, normalidade, heterogeneidade de variâncias.

\section{ABSTRACT}

\section{EFFECTS OF TRANSFORMATIONS OF DATA ON THE COEFFICIENT OF VARIATION}

Transformation of data is an auxiliary process used with the analysis of variance to turn it valid when one or more of the basic hypotheses could not be verified. Although, even if calculated correctly, it can bring the inconvenient of change in the analyzed parameters. The coefficient of variation (CV), is one of these parameters and presents as disadvantage the great sensibility to transformations of the data. In this work was studied the effect of power transformations from Tukey Transformations Scale, on CV, for 110 experiments of upland rice, classified by trial type and sow regions, through the variable yield and its transformations. The Lilliefors and Cochran tests were used to assess the normal distribution of the data and the variance homogeneity of the treatments. The most efficient transformations were the square root and the logarithmns, both reducing the values of $\mathrm{CV}$, correcting the lack of normality and/or variance heterogeneity when transformations were needed. In general, the experiments with small CV were those from the Advanced Comparative Trial group together with those from Central region.

Key words: transformation of data, coefficient of variation, upland rice, normality, heterogeneity of variances. 


\section{INTRODUÇÃO}

A análise da variância é uma técnica estatística desenvolvida por Fisher (1925) para sistematizar e facilitar a análise e interpretação de dados experimentais. Para que a análise e os testes de significância aplicados tenham validade, necessita-se que as condições básicas da análise de variância sejam atendidas. Há um consenso generalizado de que quando isso não ocorre, a solução mais recomendada é a transformação dos dados originais (Cochran, 1947; Mead \& Curnow, 1983; Gomez \& Gomez, 1983).

Geralmente o pesquisador sabe historicamente quais variáveis precisam ser transformadas, por isso, muitas vezes aplicar transformações conhecidas antes de qualquer inspeção prévia dos dados. Fisher \& Vanbelle (1983) recomendam que não se façam transformações desnecessárias e que se tenha muito cuidado, particularmente com estimações. Portanto, ao empregar uma transformação deve-se ter uma razão lógica, o que nem sempre ocorre, levando às vezes a utilizar transformações indiscriminadamente, sem nenhum critério ou preocupação de sua validade. Assim ao considerar-se uma transformação, deve-se verificar se os benefícios justificam o esforço necessário e a inconveniência que ela pode causar.

As transformações raiz quadrada, logarítmica, angular e inversa têm sido aplicadas a diferentes tipos de dados, para observação de seus efeitos sobre os resultados da análise de variância, e só devem ser empregadas se os dados violarem as suposições de normalidade e homogeneidade de variância (Bartlett, 1947; Demétrio, 1978 e Bowman \& Watson, 1997). Quando não há razão teórica para se escolher uma transformação específica, Mead \& Curnow (1983) sugerem ao experimentador selecionar uma transformação empiricamente e discutem como escolher a melhor transformação. Sokal \& Rohlf (1995) recomendam as transformações Box-Cox em vez de tentar várias transformações para descobrir a melhor delas. Box \& Cox (1964) desenvolveram um método para escolher a melhor transformação dentro da 
família de transformações de potência. Uma discussão sobre esta família de transformações é apresentada em Emerson \& Stoto (1983). Baseando-se também na família de transformações de potência, Tukey (1977) propôs uma Escala de Transformações, eficiente na maioria dos casos.

Após a análise dos dados transformados, devem-se verificar com precaução os resultados obtidos, pois, parâmetros, como o coeficiente de variação $(\mathrm{CV})$, o coeficiente de determinação $\left(\mathrm{R}^{2}\right)$, o quadrado médio do resíduo (QMR), além de testes aplicados, podem ser influenciados conforme o tipo de transformação usada. Dentre estes, o coeficiente de variação, é uma medida de variação relativa e apresenta, como desvantagem, grande sensibilidade às transformações de dados. Por isso alguns autores o consideram de uso limitado, embora transformações possam resultar em sua melhoria. Segundo Sampaio (1998) a presença de CV's muito altos, pode ser percebida em situações onde a análise de variância não tem suas hipóteses garantidas e, na maioria das vezes, as variáveis que demandam transformação apresentam alterações, responsáveis pela elevação do CV. Em estudo recente sobre o desempenho de medidas de validade, Bowman \& Watson (1997), examinaram os, efeitos de transformações de dados sobre o $\mathrm{CV}$ e $\mathrm{R}^{2}$ com dados simulados e concluíram que o $\mathrm{R}^{2}$ pode ser uma medida estatística alternativa para avaliar o desempenho de experimentos.

Pouca ou quase nenhuma atenção tem sido reservada ao estudo do efeito das transformações sobre o coeficiente de variação. $O$ que vem sendo estudado são critérios de classificação do $C V$ para experimentos agrícolas, como pode ser visto em Steel \& Torrie (1980), Pimentel-Gomes (1985), Estefanel et al. (1987), Garcia (1989) e mais recentemente em SCAPIM et al. (1995) e Amaral et al. (1997). O presente trabalho teve por objetivo verificar os efeitos das transformações sobre os resultados obtidos com o coeficiente de variação, com dados da produção de grãos do arroz de sequeiro. 
MATERIAL E MÉTODOS

Material

Foram utilizados, para análise, dados de 110 experimentos relativos a produção de grãos ( $\mathrm{kg} / \mathrm{ha}$ ) do arroz de sequeiro, cedidos pela Embrapa Arroz e Feijão. Esses experimentos foram conduzidos no período de 1991 a 1998, classificados de acordo com o tipo de ensaio (Ensaio Comparativo Avançado-ECA, Ensaio Comparativo Preliminar-ECP, MeIhoramento Populacional, Ensaio de Densidade Populacional e Outros) e a região de cultivo, Norte (AC, $\mathrm{AM}, \mathrm{AP}, \mathrm{RO}$ ); Nordeste (MA, PI, CE) e Centro-Oeste (GO, MT, MS, TO, MG). Os experimentos não foram realizados especialmente para atender os objetivos deste trabalho. Para efeito deste estudo, foram agrupados de acordo com a Tabela 1.

Tabela 1. Distribuição dos experimentos de acordo com o tipo de ensaio e região de cultivo

\begin{tabular}{c|c}
\hline Grupos & Número de experimentos \\
\hline Geral & 110 \\
- Regiões & \\
Centro-Oeste & 78 \\
Outras & 32 \\
- Ensaios & \\
ECA & 56 \\
ECP & 30 \\
Outros & 24 \\
\hline
\end{tabular}

Métodos

Considerando a natureza da variável (x) analisada (produção de grãos), foram aplicadas transformações de potência aos dados originais, usando uma modificação da Escala de Transformação de Tukey (1977). Obtiveram-se seis variáveis para análise; $\mathrm{P} 1-\log$ aritmo de $\mathrm{x} ; \mathrm{P} 2-\sqrt{x} ; \mathrm{P} 3-\mathrm{x}^{2} ; \mathrm{P} 4-\mathrm{x}^{-1}$ ; P5 - $\mathrm{x}^{-1 / 2}$; e P6 $-\mathrm{x}^{-2}$ (Figura 1). A partir destas, além de obter as estimativas do coeficiente de variação, avaliaram-se as condições de normalidade e 
homogeneidade de variância dos dados, através dos testes de Lilliefors e Cochran, para interpretação dos efeitos das transformações de dados sobre o $\mathrm{CV}$, independente da necessidade ou não de uma transformação (Figura 1).

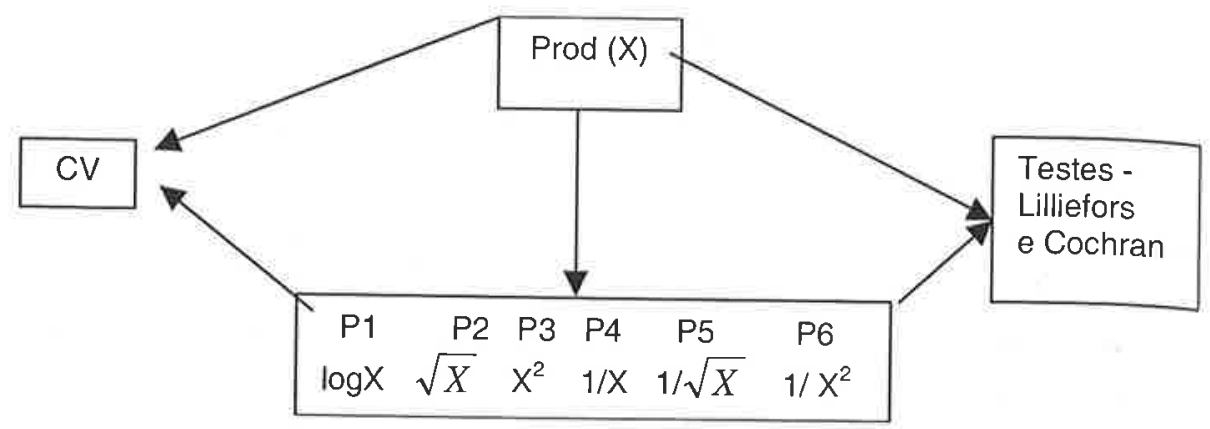

Figura 1. Esquema dos métodos utilizados. Prod = Produção.

\section{RESULTADOS E DISCUSSÃO}

Os resultados obtidos são apresentados, primeiro em termos gerais e depois classificados de acordo com a região de cultivo (CentroOeste e Outras) e o tipo de ensaio (ECA, ECP e Outros), para a variável produção de grãos e suas transformações.

As estimativas da média do CV são apresentadas na Tabela 2, e baseando-se no critério de classificação do CV de Pimentel-Gomes (1985), para a variável sem transformação x (produção), classificam-se em altas, e para as transformadas em baixas, médias e muito altas.

A acentuada variação entre as estimativas para as transformações e a variável original (Tabela 2), ocorreu devido aos tipos de transformações de potência $\left(x^{p}\right)$ empregados. Percebeu-se uma diminuição nas estimativas do $C V$ após as transformações com p variando entre - $1 \frac{1}{2}$ e $1 \frac{1}{2}$; estas transformações reduziram a amplitude e a variação entre os dados. Por outro lado, verificou-se uma elevação das estimativas do CV após as transformações com $\mathrm{p}$ $\leq-1$ ou $p=2$, as transformações inversas reduziram a amplitude de variação 
Tabela 2. Estatísticas descritivas do coeficiente de variação (CV), de acordo com o grupo, para cada uma das variáveis antes e após as transformações.

\begin{tabular}{|c|c|c|c|c|c|c|c|c|}
\hline \multirow{2}{*}{ Grupo } & \multirow{2}{*}{$\begin{array}{l}\text { Estatísticas } \\
\text { do CV }(\%)\end{array}$} & \multicolumn{7}{|c|}{ Variáveis } \\
\hline & & PROD & P1 & $\mathrm{P} 2$ & P3 & P4 & P5 & $\mathrm{P6}$ \\
\hline \multirow{4}{*}{$\begin{array}{l}\frac{1}{4} \\
\frac{1}{山} \\
\frac{1}{5}\end{array}$} & Média & 21,14 & 3,65 & 11,34 & 40,39 & 43,41 & 16,01 & 111,45 \\
\hline & Desvio Padrão & 9,21 & 2,67 & 5,45 & 17,48 & 63,07 & 16,18 & 130,80 \\
\hline & Máximo & 51,36 & 19,13 & 35,17 & 95,17 & 434,98 & 139,13 & 718,36 \\
\hline & Mínimo & 3,78 & 0,52 & 1,90 & 7,75 & 4,21 & 2,00 & 9,47 \\
\hline \multirow{4}{*}{ 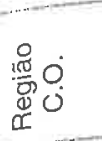 } & Média & 20,05 & 3,62 & 10,88 & 38,08 & 46,60 & 16,36 & 115,56 \\
\hline & Desvio Padrão & 9,30 & 3,01 & 5,72 & 17,21 & 73,33 & 18,63 & 143,90 \\
\hline & Máximo & 51,36 & 19,13 & 35,17 & 95,17 & 434,98 & 139,13 & 718,36 \\
\hline & Mínimo & 3,78 & 0,52 & 1,90 & 7,75 & 4,21 & 2,00 & 9,47 \\
\hline \multirow{4}{*}{ 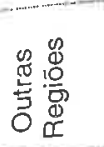 } & Média & 23,80 & 3,74 & 12,47 & 46,04 & 35,63 & 15,16 & 101,43 \\
\hline & Desvio Padrão & 8,40 & 1,56 & 4,54 & 16,83 & 21,97 & 7,26 & 90,52 \\
\hline & Máximo & 43,30 & 7,49 & 23,03 & 87,91 & 103,25 & 36,57 & 449,38 \\
\hline & Mínimo & 12,22 & 1,77 & 6,27 & 23,79 & 14,26 & 6,77 & 31,22 \\
\hline \multirow{4}{*}{ 志 } & Média & 19,53 & 2,85 & 10,15 & 37,92 & 27,92 & 11,99 & 78,73 \\
\hline & Desvio Padrão & 7,30 & 1,24 & 3,81 & 14,77 & 17,11 & 5,57 & 73,66 \\
\hline & Máximo & 40,60 & 6,26 & 19,60 & 88,13 & 101,37 & 29,99 & 449,38 \\
\hline & Mínimo & 3,78 & 0,52 & 1,90 & 7,75 & 4,21 & 2,00 & 9,47 \\
\hline \multirow{4}{*}{ 号 } & Média & 22,20 & 3,54 & 11,57 & 43,49 & 33,10 & 14,07 & 94,29 \\
\hline & Desvio Padrão & 10,12 & 1,89 & 5,45 & 20,09 & 21,73 & 7,92 & 80,66 \\
\hline & Máximo & 46,90 & 7,99 & 24,27 & 95,17 & 103,25 & 36,57 & 346,04 \\
\hline & Mínimo & 6,62 & 0,84 & 3,25 & 13,88 & 6,66 & 3,24 & 15,75 \\
\hline \multirow{4}{*}{ 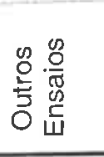 } & Média & 23,59 & 5,66 & 13,85 & 42,30 & 92,44 & 27,80 & 209,26 \\
\hline & Desvio Padrāo & 11,31 & 4,46 & 7,62 & 19,28 & 120,27 & 30,09 & 217,13 \\
\hline & Máximo & 51,36 & 19,13 & 35,17 & 83,57 & 434,98 & 139,13 & 718,36 \\
\hline & Mínimo & 11,07 & 1,37 & 5,31 & 19,89 & 11,34 & 5,54 & 23,90 \\
\hline
\end{tabular}

PROD - produção; P1 - logaritmo da produção; P2 - raiz quadrada da produção; P3 quadrado da produção; P4 - inverso da produção; P5 - inverso da raiz quadrada da produção; P6 - inverso do quadrado da produção.

dos dados, e tornaram as médias das variáveis muito pequenas, enquanto que a transformação $\mathrm{x}^{2}$ aumentou a amplitude e a média dos dados, afetando muito as estimativas do CV nestes casos. Essa sensibilidade do CV a transformações de dados, podendo ser elevado ou reduzido, de acordo com a transformação aplicada, confirma os resultados de Mandel (1964) e Bowman \& Watson (1997). As transformações que produziram a maior redução do CV 
foram $\sqrt{x}, \log \mathrm{x} \mathrm{e}^{-1 / 2}$ (Tabela 2), concordando com Tukey (1977) que considerou estas transformações como as mais importantes de sua Escala.

Em relação aos grupos analisados, poucas diferenças foram observadas nas estimativas dos $\mathrm{CV}$, entretanto, maiores diferenças foram percebidas entre as variáveis analisadas, como em Estefanel et al. (1987). Deve-se ressaltar que se obtiveram estimativas de $\mathrm{CV}$ menores, no grupo ECA e região Centro-Oeste (Tabela 2), o que pode ser explicado pelo fato do ECA ser a etapa final do processo de seleção de linhagens, e por serem os Estados da região Centro-Oeste mais apropriados para o cultivo do arroz, no sistema de sequeiro, principalmente pela condição climática favorável (Ferreira et al. 1983).

Maiores detalhes dos efeitos das transformações sobre o CV são observados, considerando as hipóteses de normalidade da distribuição dos dados e homogeneidade de variância. Desta forma, os resultados são apresentados para quando as duas condições foram atendidas, uma delas foi atendida, as duas não foram atendidas (Tabelas 3 e 4) e pelo menos uma não foi atendida (Tabela 5), em relação à variável original.

A Tabela 3 apresenta o número de experimentos em que as hipóteses de normalidade da distribuição dos dados e homogeneidade de variância são atendidas para variável PROD. Aplicadas as transformações os resultados tornaram-se desfavoráveis, mostrando com isso que pode haver mudanças nas conclusões se for feita uma transformação desnecessária. Verificaram-se menos influências sobre a normalidade dos dados e a homogeneidade de variância com aplicação das transformações raiz quadrada, quadrado e logarítmica respectivamente.

Como se pode notar na Tabela 3 , foram poucos os casos de experimentos que atendiam à condição de normalidade e não atendiam à de homogeneidade de variância. Essa baixa frequiência de experimentos pode ser devida ao emprego correto das técnicas experimentais e condições apropriadas para cultivo. Neste caso, ocorre heterogeneidade entre os tratamentos, que talvez possa ser denominada irregular (Gomez \& Gomez, 1983; Banzatto \& Kronka, 1989). Os métodos mais apropriados e indica- 


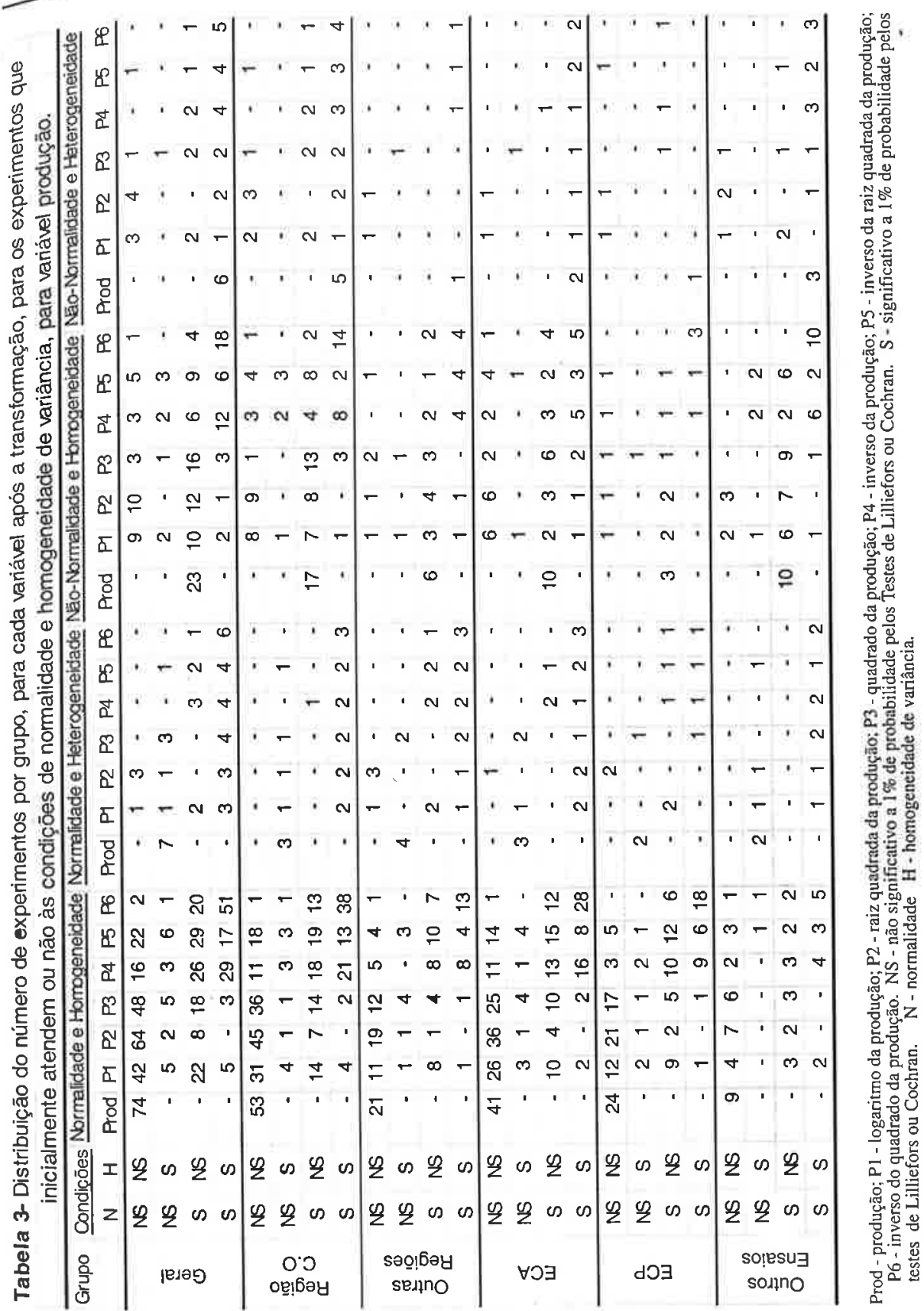


dos para este tipo de falha são a subdivisão da variância residual ou a eliminação de parte do experimento (Cochran, 1947; Gomez \& Gomez, 1983). Após as transformações ocorreu uma pequena melhora dos resultados, sendo que a melhor transformação foi a raiz quadrada $(\sqrt{x})$, e a pior, o inverso do quadrado $\left(\mathrm{x}^{-2}\right)$. Isso pode ser um indicativo de que a transformação de dados não é a técnica mais indicada, talvez um método não-paramétrico seja mais adequado nestes casos (Conover, 1980). Sabese que o valor do teste $\mathrm{F}$ da análise de variância, é afetado pela heterogeneidade de variância associada a dados provenientes da distribuição normal (Cochran, 1947; Zimmermann, 1987).

Em relação aos experimentos originalmente com não-normalidade e homogeneidade de variância (Tabela 3), considerando o controle das condições, as transformações apresentaram-se bem mais eficientes que o caso anterior; sendo a raiz quadrada e a logarítmica, as melhores, e o inverso do quadrado, a pior. Essa situação é menos importante, pois a robustez do teste F, é menos afetada se acompanhada de homogeneidade de variância Zimmermann (1987) mostrou que o tamanho e o poder do teste $F$, não são afetados quando os dados têm variância homogênea independentemente da distribuição de probabilidade.

Também foram analisados os experimentos que não atendiam às condições de normalidade e homogeneidade de variância (Tabela 3), denominada heterogeneidade regular (Gomez \& Gomez, 1983; Banzatto \& Kronka, 1989). Verificou-se nessa situação que as transformações logarítmica e raiz quadrada foram as mais eficientes, concordando com Cochran (1947) e Gomez \& Gomez (1983), que afirmam que a aplicação de uma transformação adequada, quando a heterogeneidade é regular produz bons resultados.

Quando as condições de normalidade e homogeneidade de variância são atendidas, e aplica-se uma transformação indiscriminadamente, observam-se modificações desfavoráveis tanto na forma da distribuição dos dados, como em relação à homogeneidade de variância. Mudanças menores são observadas após a transformação raiz quadrada, mas 
após a transformação inverso do quadrado, verificaram-se mudanças drásticas dos experimentos que atendiam às duas condições. Por outro lado, quando pelo menos uma das condições não é atendida, após as transformações percebe-se uma melhoria dos resultados, mas em todos os casos grande parte dos experimentos continuaram a não atender pelo menos a uma das condições, especialmente após as transformações inversas. As transformações mais eficientes foram a raiz quadrada e a logarítmica, a transformação raiz quadrada reduziu as estimativas do CV e aumentou o número de experimentos que atendiam às duas condições para todos os grupos (Tabela 5). Com isso, observou-se que transformações de dados só devem ser aplicadas, se uma ou mais das hipóteses da análise de variância não são satisfeitas. Isto confirma que, quando as condições da análise de variância estiverem satisfeitas, deve-se evitar a transformação dos dados, não só por questões práticas, mas também por inversões na ordenação das médias que ela pode trazer, como em Demétrio (1978).

Em relação às estimativas da média dos $\mathrm{CV}$, os experimentos que atendiam às duas condições apresentaram médias inferiores aos que não atendiam à pelo menos uma condição, mesmo após as transformações (Tabelas 4 e 5). Situações onde a análise de variância fica inviabilizada, se denunciam por apresentarem coeficientes de variação muito elevados (Sampaio, 1998). Destaca-se uma grande diferença das estimativas das médias dos CV entre as duas situações, principalmente, após a transformação inverso do quadrado (Tabela 5). Isto confirma novamente que as estimativas dos $\mathrm{CV}$ apresentaram-se extremamente sensíveis à transformações.

Em torno de $65 \%$ a $80 \%$ dos experimentos analisados, de acordo com o grupo, tiveram as duas condições atendidas, exceto o grupo Outros Ensaios. Neste caso, a aplicação de qualquer das transformações de potência mostrou inadequada. Para os outros casos, onde pelo menos uma condição não é atendida, as transformações foram mais eficientes em experimentos que tinham heterogeneidade regular e não-normalidade associada a homogeneidade de variância, e menos eficientes em experimentos com normalidade e heterogeneidade de variância, recomendando-se um controle maior das cultivares e da região de cultivo. 


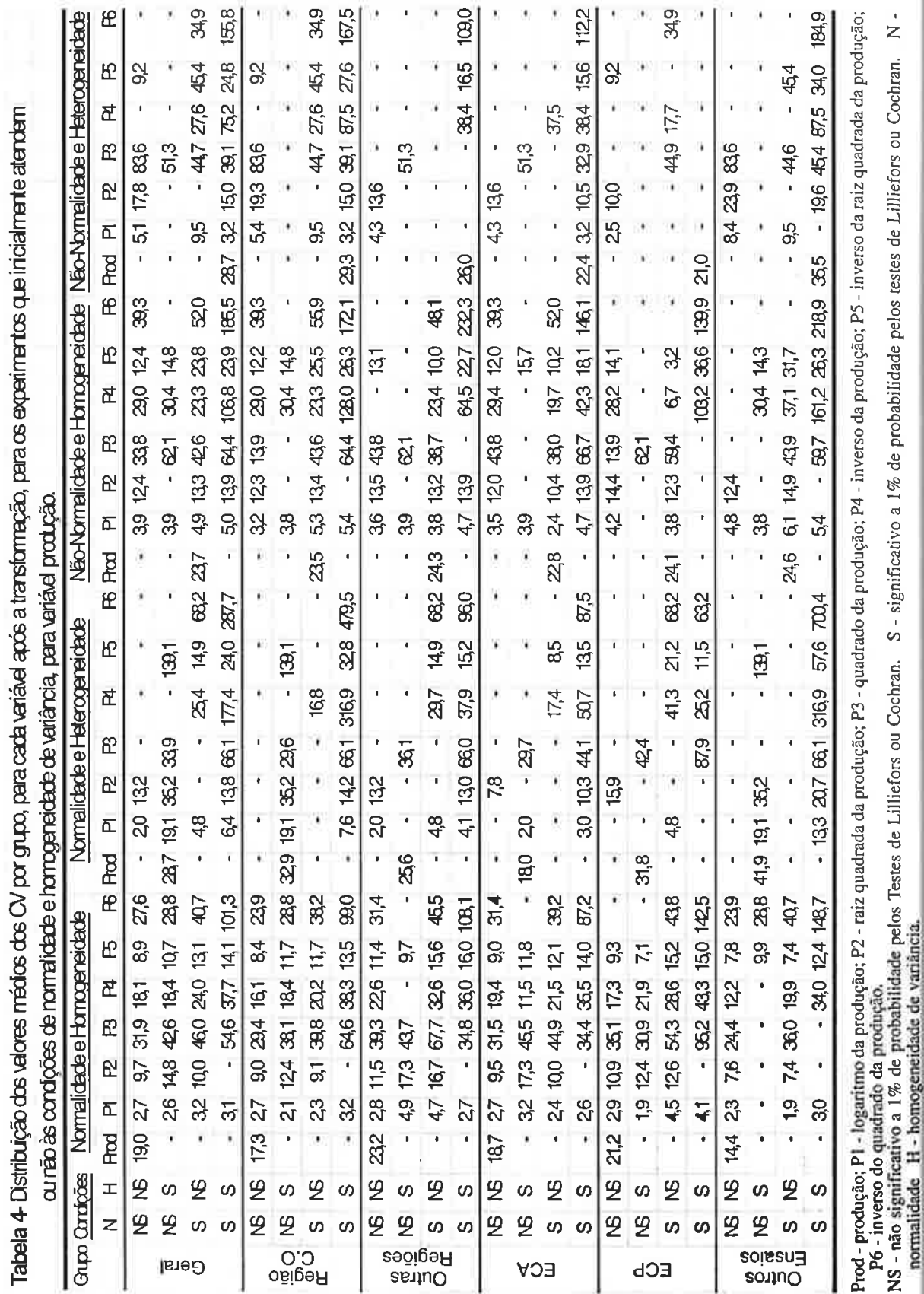


Tabela 5. Distribuição dos valores médios dos CV e do número de experimentos por grupo, para cada variável após a transformação, para os experimentos que inicialmente atendem as condições de normalidade e homogeneidade de variância e para os que não atendem a pelo menos uma condição, para variável produção.

\begin{tabular}{|c|c|c|c|c|c|c|c|c|c|}
\hline \multirow[t]{2}{*}{ Grupo } & \multirow{2}{*}{\multicolumn{2}{|c|}{$\begin{array}{l}\text { Condições e } \\
\text { Resultados }\end{array}$}} & \multicolumn{7}{|c|}{ Variáveis } \\
\hline & & & PROD & P1 & P2 & P3 & P4 & P5 & P6 \\
\hline \multirow{6}{*}{ 売 } & Atende às 2 & NS & 74 & 42 & 64 & 48 & 16 & 22 & 2 \\
\hline & condiçōes & s & 0 & & 10 & 26 & 58 & 52 & 72 \\
\hline & & Média(\%) & 19,00 & 2,88 & 9,90 & 36,97 & 27,88 & 11,87 & 81,95 \\
\hline & Nāo atende à & NS & 0 & 13 & 17 & 4 & 3 & 6 & 1 \\
\hline & pelo menos 1 & $\mathrm{~S}$ & 36 & 23 & 19 & 32 & 33 & 30 & 35 \\
\hline & ondição & Média(\%) & 25,55 & 5,24 & 14,32 & 47,43 & 75,32 & 24,51 & 172,09 \\
\hline \multirow{5}{*}{ 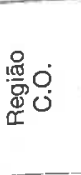 } & Atende às 2 & NS & 53 & 31 & 45 & 36 & 11 & 18 & 1 \\
\hline & condiçōes & & 0 & 22 & 8 & 17 & 42 & 25 & 52 \\
\hline & & Medi & 17,34 & 2.59 & $\frac{9,05}{12}$ & $\frac{33,65}{2}$ & $\frac{26,44}{3}$ & $\frac{11,02}{5}$ & $\frac{81,31}{1}$ \\
\hline & Nao atende a & NS & $\begin{array}{r}0 \\
05\end{array}$ & 10 & $\begin{array}{l}12 \\
13\end{array}$ & 23 & 22 & 20 & 24 \\
\hline & condiçāo & Média(\%) & 25,82 & 5,80 & 14,77 & 47,46 & 89,33 & 27,67 & 188,18 \\
\hline \multirow{5}{*}{ 预 } & Atende às 2 & NS & 21 & 11 & $\overline{9}$ & 12 & 5 & 4 & 1 \\
\hline & condiçōes & & 0 & 10 & 2 & 9 & 16 & 17 & 20 \\
\hline & Näo atende à & $\frac{\text { Med }}{\text { NS }}$ & $\frac{23,21}{0}$ & $\frac{3,62}{3}$ & $\frac{12,04}{5}$ & $\frac{45,34}{2}$ & $\frac{31,52}{-}$ & $\frac{14,00}{1}$ & \\
\hline & pelo menos 1 & $s$ & 11 & 8 & 6 & 9 & 11 & 10 & 11 \\
\hline & condiçāo & Média(\%) & 24,93 & 3,97 & 13,29 & 47,38 & 43,49 & 17,34 & 135,52 \\
\hline \multirow{6}{*}{ 古 } & Atende às 2 & NS & 41 & 26 & 36 & 25 & 11 & 14 & 1 \\
\hline & condiç̧̄es & S & 0 & 15 & 5 & 16 & 30 & 27 & 40 \\
\hline & & Média(\%) & 18,71 & 2,68 & 9,72 & 36,27 & 26,17 & 11,38 & 71,80 \\
\hline & Nāo atende à & NS & 0 & 7 & 8 & 2 & 2 & 4 & 1 \\
\hline & pelo menos 1 & $s$ & 15 & 8 & 7 & 13 & 13 & 11 & 14 \\
\hline & condicão & Média(\%) & 21,77 & 3,31 & 11,33 & 42,43 & 32,70 & 13,67 & 97,65 \\
\hline \multirow{6}{*}{ 总 } & Atende às 2 & NS & 24 & 12 & 21 & 17 & 3 & 5 & - \\
\hline & condiçōes & $S$ & 0 & 12 & 3 & 7 & 21 & 19 & 24 \\
\hline & & Média(\%) & 21,22 & 3,43 & 11,09 & 41,42 & 32,11 & 13,59 & 93,45 \\
\hline & Nāo atende à & NS & 0 & 2 & 4 & 1 & 1 & 2 & - \\
\hline & pelo menos 1 & s & 6 & 4 & 2 & 5 & 5 & 4 & 6 \\
\hline & condição & Média(\%) & 26,12 & 3,98 & 13,48 & 51,76 & 37,07 & 15,97 & 97,66 \\
\hline \multirow{5}{*}{ 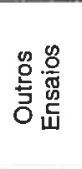 } & Atende às 2 & NS & 9 & 4 & 7 & 6 & 2 & 3 & 1 \\
\hline & condiçōes & S & 0 & 5 & 2 & 3 & 7 & 6 & 8 \\
\hline & & Média(\% & 14,42 & 2,32 & 7,52 & 28,30 & 24,41 & 9.51 & 97,53 \\
\hline & Não atende à & NS & 0 & 3 & 5 & 1 & - & - & - \\
\hline & pelo menos 1 & $\begin{array}{l}\text { S } \\
\text { Média(\%) }\end{array}$ & 15 & 12 & $\begin{array}{l}10 \\
1765\end{array}$ & $\begin{array}{l}14 \\
50.71\end{array}$ & $\begin{array}{c}15 \\
133.25\end{array}$ & $\begin{array}{l}15 \\
38.78\end{array}$ & $\begin{array}{c}15 \\
276.30\end{array}$ \\
\hline
\end{tabular}

PROD - produção; P1 - logaritmo da produção; P2 - raiz quadrada da produção; P3 quadrado da produção; P4 - inverso da produção; P5 - inverso da raiz quadrada da produção; P6 - inverso do quadrado da produção.

NS - não significativo a $1 \%$ de probabilidade pelos Testes de Lilliefors e Cochran, para as duas condições.

S - significativo a $1 \%$ de probabilidade pelos Testes de Lilliefors e Cochran, para pelo menos uma condição. 


\section{CONCLUSÕES}

1. As transformações logarítmica $(\log x)$, raiz quadrada $(\sqrt{x})$ e inverso da raiz $\left(\mathrm{x}^{-1 / 2}\right)$, reduzem os valores do $\mathrm{CV}$, enquanto que as transformações quadrado $\left(\mathrm{x}^{2}\right)$, inverso $\left(\mathrm{x}^{-1}\right)$ e inverso do quadrado $\left(\mathrm{x}^{-2}\right)$ os elevaram.

2. A aplicação de qualquer uma das transformações de potência, para experimentos que atendem as condições de normalidade dos dados e homogeneidade de variância, pode tornar inválida uma dessas condições.

3. As transformações mais eficientes foram a raiz quadrada e a logarítmica, que além de reduzir os valores do $\mathrm{CV}$, corrigiram a falta de normalidade dos dados e/ou a heterogeneidade de variância, quando havia necessidade de transformação.

4. A aplicação da transformação raiz quadrada é a que tem menos probabilidade de gerar resultados inadequados, quando se percebe, através de rápida inspeção dos dados, que uma das hipóteses da análise de variância não se verifica.

5. A transformação raiz quadrada é a que menos altera os resultados, quando se aplica uma transformação indiscriminadamente.

\section{REFERÊNCIAS}

AMARAL, A.M;; J.A. MUNIZ \& M. SOUZA., 1997. Avaliação do Coeficiente de Variação como Medida de Precisão na Experimentação com Citros. Pesquisa Agropecuária Brasileira, 32 (12): 1221-1225.

BANZATO, D.A. \& S.N. KRONKA., 1989. Experimentação Agrícola. Jaboticabal: Funep.

BARTLETT, M.S., 1947. The Uses of Transformations. Biometrics, 3 : $39-52$. 
BOWMAN, D.T. \& C. WATSON., 1997. Measures of Validity in Culti-

var Performance Trials. Agronomy Journal, 89 (6): 860-866. BOX, G.E.P. \& D.R. COX., 1964. An Analysis of Transformations.

Journal of the Royal Statistical Society. Séries B, 26: 211-252.

COCHRAN, W.G., 1947. Some Consequences When the Assumptions

for the Analysis of Variance are not Satisfied. Biometrics, 3: 22-38. CONOVER, W.J., 1980. Practical Nonparametric Statistics. New York:

John Wiley.

DEMÉTRIO, C.G.B., 1978. Transformação de Dados. Efeitos sobre a Análise de Variância. Piracicaba, ESALQ/USP. (Dissertação de Mestrado).

EMERSON, J.D. \& M.A. STOTO., 1983. Understanding Robust and

Exploratory Data Analysis. New York: John Wiley.

ESTEFANEL, V.; I.A.B. PIGNATÁRO \& L. STORCK., 1987. Avaliação

do Coeficiente de Variação de Experimentos com Algumas Culturas Agrícolas. In: SIMPÓSIO DE ESTATÍSTICA APLICADA À EXPERIMENTAÇÃO AGRONÔMICA, 2. Londrina. Anais ... Londrina: Univ. Est. de Londrina/Reg. Bras. da Soc. Intern. de Biometria.

FERREIRA, M. E.; T. YAMADA \& E. MALAVOLTA., 1983. Cultura do Arroz de Sequeiro. Piracicaba, Inst. Potassa.

FISHER, R.A., 1925. Statistical Methods for Research Workers.

Edingurgh: Oliver \& Boyd.

FISHER, L.D. \& G. VANBELLE., 1993. Biostatistics. A Methodology

for the Hearth Sciences. Jonh Wiley.

GARCIA, C.H., 1989. Tabelas para Classificação do Coeficiente de Variação. Piracicaba: Instituto de Pesquisas e Estudos Florestais. 12 p. Circular Técnica, 171.

GOMEZ, K.A. \& A.A. GOMEZ., 1983. Statistical Procedures for Agricultural Research. New York: John Wiley. MANDEL, J., 1964. The Statistical Analysis of Experimental Data.

New York: Dover Publications.

MEAD, R. \& R.N. CURNOW., 1983. Statistical Methods in Agriculture and Experimental Biology. New York: Chapman and Hall. 
PIMENTEL-GOMES, F., 2000. Curso de Estatística Experimental. Piracicaba. $14^{\mathrm{a}}$ ed., Piracicaba.

SAMPAIO, I.B.M., 1998. Estatística Aplicada à Experimentação Animal. Fundação de Ensino e Pesq. em Medicina Veterinária e Zootecnia. Depto de Zootecnia, UFMG.

SCAPIM, C.A.; C.G.P. CARVALHO. \& C.D. CRUZ., 1995. Uma Proposta de Classificação dos Coeficientes de Variação para Cultura do Milho. Pesquisa Agropecuária Brasileira, 30 (5): 683-686.

SOKAL, R.R. \& F.J. ROHLF., 1995. Biometry. $3^{\mathrm{a}}$ ed. New York: W. H.

Freeman and Company.

STEEL, R.G.D. \& J.H. TORRIE., 1980. Principles and Procedures of Statistics. A Biometrical Approach. $2^{\text {a }}$ edição, New York: McGrawHill Book.

TUKEY, J.W., 1977. Exploratory Data Analysis. Addison-Wesley Publ. Co., Reading. Mass.

ZIMMERMANN, F.J.P., 1987. Efeito de Heterogeneidade de Variância e Distribuição de Probabilidade dos Dados sobre o Poder e Tamanho do Teste F. Pesquisa Agropecuária Brasileira, 22 (11/12): 12091213. 